\title{
Assessment of Inferior Thyroid Artery by Color Doppler Ultrasound in Diffuse Toxic Goiter- Experience at NINMAS
}

\author{
${ }^{1}$ Nasreen Sultana, ${ }^{1}$ Sharmin Quddus, ${ }^{1}$ Rahima Perveen, ${ }^{1}$ Mohammad Simoon Salekin, ${ }^{2}$ Md. Bashir, and ${ }^{1}$ Nurun Nahar

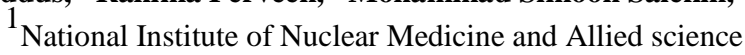 \\ ${ }^{2}$ Dhaka Medical College
}

Correspondence Address: Dr. Nasreen Sultana MD, Associate Professor. NINMAS, BAEC. Dhaka, e-mail: nasreeninm@yahoo.com

\begin{abstract}
Objective: The aim of this study was to compare the B mode ultrasonography findings of thyroid gland and peak systolic velocity of inferior thyroid artery by color Doppler application, in patients with diffuse toxic goiter at the time of diagnosis and 6-8 months after radioiodine 131-I therapy.

Patients and Methods: This was a prospective observational study conducted in National Institute of Nuclear Medicine \& Allied Sciences (NINMAS) between June 2015 and October 2016. Forty consecutive diagnosed diffuse toxic goiter patients were selected at the time of diagnosis prior to radioiodine therapy. An ultrasonography evaluation of thyroid gland (measurement of anterior posterior diameter for convenience) and measurement of peak systolic velocity of inferior thyroid artery in both sides of thyroid gland were performed before and after 131-I therapy (6-8 months). The examination was performed in thyroid division of NINMAS by advanced ultrasound machine with a linear transducer (5-12 MHz). The patients were divided into two groups. Group A-diffuse toxic goiter prior to radioiodine therapy and group B- same patients 6-8 months after radioiodine therapy.
\end{abstract}

Results: A reduction in the anterior -posterior diameter of the thyroid gland was observed from pre to post treatment evaluation, performed at 6 and or 8 months after radioiodine therapy. Color Doppler showed increased parenchymal vascularity at the time of diagnosis which gradually declined after radioiodine therapy. Thyroid blood flow, as assessed by PSV of ITA, was significantly higher in Group A than in Group B respectively $(70.5 \pm 15.5 \mathrm{~cm} / \mathrm{sec}$ versus $22.2 \pm 12.9 \mathrm{~cm} / \mathrm{sec})$. PSV was measured in 10 healthy euthyroid adults \& their PSV ranged $13-20 \mathrm{~cm} / \mathrm{Sec}$. Parenchymal vascularity of the thyroid gland was higher in hyperthyroid patients than in euthyroid patients

Conclusion: Assessment of thyroid gland by B-mode ultrasound and blood flow by color Doppler study (CDS) is an effective marker in the initial diagnosis of diffuse toxic goiter.

Key words: Thyroid Doppler, Inferior Thyroid Artery, Diffuse Toxic Goiter.

\section{INTRODUCTION}

Diffuse toxic goiter is an autoimmune, diffuse, chronic disease of thyroid gland with evidence of genetic predisposition and unknown etiology. It is characterized by markedly increased the size of thyroid gland and also blood flow of the gland (1). Increased thyroidal blood flow is seen to be correlated with iodine uptake and thyroid hormone levels $(2,3)$. The disease is characterized by one or more changes: hyperthyroidism, goiter, ophthalmopathy, skin changes and pretibial myxedema. Diffuse toxic goiter patients can have a stable remission after medical therapy or can have relapsing course. Radioactive iodine treats hyperthyroidism by gradually shrinking thyroid gland ultimately destroying the gland. Unlike anti-thyroid medications, radioactive iodine is a permanent and more reliable cure for hyperthyroidism.

99mTc pertechnetate scan and TSH receptor anti-body levels help in etiological differentiation of thyrotoxicosis in difficult situations (4). One of the most relevant clinical practice aspects in diffuse toxic patients management is to distinguish Graves' Disease(GD) in initial phase, from other types of destructive thyrotoxicosis, in addition to evaluate therapeutic methods and efficient follow-up, as well as predict early recurrence or remission of disease. Various factors like gland size, autoantibody titer, and antithyroid drug requirement are used to predict remission of GD (5). Ultrasonography is a very informative tool and color Doppler gives an additional advantage of directly measuring the tissue vascularity (6). Color flow Doppler 
sonography of the thyroid gland helps in assessing thyroid gland functional status indirectly by studying the vascularity (7). Peak systolic velocity (PSV) obtained from the inferior thyroid artery (ITA) is the parameter commonly employed for the quantitative Doppler evaluation of thyroid gland. Other estimation of thyroid blood flow is vascularization index and high-resolution power Doppler is used. Assessment is at the same time qualitative (gland visual vascularization impression) and quantitative (inferior thyroid arteries peak systolic velocity). Now a day, with the wider popularity of physical health screening, more and more cases of atypical diffuse toxic goitre have been identified. For these reason a rapid convenient and dependable method for differentiating the causes of thyrotoxicosis especially to differentiate from thyroiditis is urgently needed. In clinical practice $99 \mathrm{mTc}$ thyroid scan is still the gold standard. However, it is not generally available and done by using ionizing radiation $(2,3)$. It can be influenced by iodine containing diet and medications. More over $99 \mathrm{mTc}$ scan cannot be used in pregnancy and lactation. On the other hand PSV of ITA is non- invasive, easily available, cost effective and useful information can rapidly be obtained in Nuclear Medicine department. It can also be done in pregnancy and lactation. Normal peak systolic velocity of ITA: $23-25 \mathrm{~cm} / \mathrm{sec}$ (2). Diffuse toxic goiter will be considered when PSV of ITA is more than 40 $\mathrm{cm} / \mathrm{sec}$ (3). In addition, this method is used in differential diagnosis with other cause of thyrotoxicosis in early stage (6-10). The objective of this study was to compare the B mode ultrasonography findings of thyroid gland and peak systolic velocity of inferior thyroid artery by color Doppler application, in patients with diffuse toxic goiter at the time of diagnosis and 6-8 months after radioiodine 131-I therapies.

\section{PATIENT AND METHODS}

Forty consecutive patients with diffuse toxic goiter and ten healthy euthyroid volunteers (age range 23-45 years; male: female $=3: 2$ ) were studied between June 2015 and October 2016 in thyroid division of NINMAS. A detailed history was taken regarding palpitations, weight loss, ophthalmic complaints and past history of similar illness or family history of thyroid illness. Thyroid function tests were done in all patients including radioiodine uptake, $99 \mathrm{mTc}$ pertechnate scan and thyroid peroxidase antibody test. Diffuse toxic goiter was diagnosed when thyrotoxicosis was associated with clinical features like weight loss or poor weight gain, palpitations, goitre, ophthalmopathy, increase free triiodothyronine (FT3), free thyroxine (FT4) level, decreased thyroid stimulating hormone (TSH) level and increased uptake on pertechnate scan $(11,12)$. The patients were subdivided into two groups for analysis: Group A - at the time of diagnosis (before radioiodine therapy) and Group $\mathrm{B}-6-8$ months after radioiodine therapy. The healthy volunteer was grouped as C. Ultrasonogram of thyroid gland and CDS were done on them.

Color Flow Doppler sonography of the thyroid gland was done in all patients using advanced ultrasound digital machine with $7.5-\mathrm{MHz}$ linear transducer. Various parameters like size (AP diameter in $\mathrm{mm}$ ), gland vascularity and peak systolic velocity (PSV) of ITA were assessed. Thyroid gland size was measured in anterior-posterior (AP) diameter for our convenience. The ITA was selected for Doppler study and flow in the thyroid artery was reported in centimeters per second from Doppler spectrum. The Doppler angle of insonation was corrected to $60^{\circ}$ or less. There was no significant difference between the PSV of inferior thyroid artery from both sides of the thyroid gland, later arithmetic mean derived from both values was taken in this study.

\section{RESULTS}

The clinical and sonographic data of the patients are summarized in Table 1. Total number of patients was forty. Male female ratio was 1:3. Mean AP diameter of thyroid gland was $38 \pm 11.2 \mathrm{~mm}$ in Group A and $20 \pm$ $3.5 \mathrm{~mm}$ in Group B. Mean peak systolic velocity (PSV) of ITA in Group A was $70.5 \pm 15.5 \mathrm{~cm} / \mathrm{sec}$ and $22.5 \pm$ $12.9 \mathrm{~cm} / \mathrm{sec}$ in Group $\mathrm{B}$, respectively. In Group $\mathrm{C}$ (healthy volunteer), PSV of ITA ranged 13-20 cm/sec with mean $\pm \mathrm{SD} 17.7 \pm 3.2 \mathrm{~cm} / \mathrm{sec}$. 


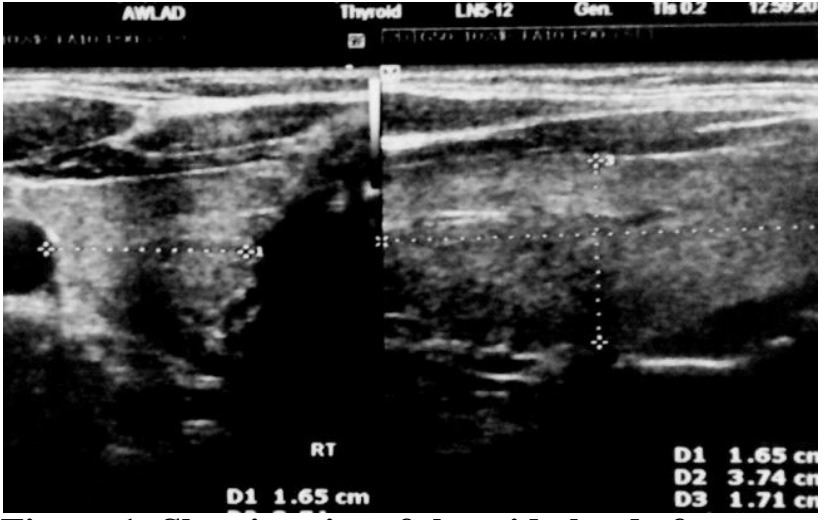

Figure 1: Showing size of thyroid gland of a patient of Group A (before radioiodine therapy).

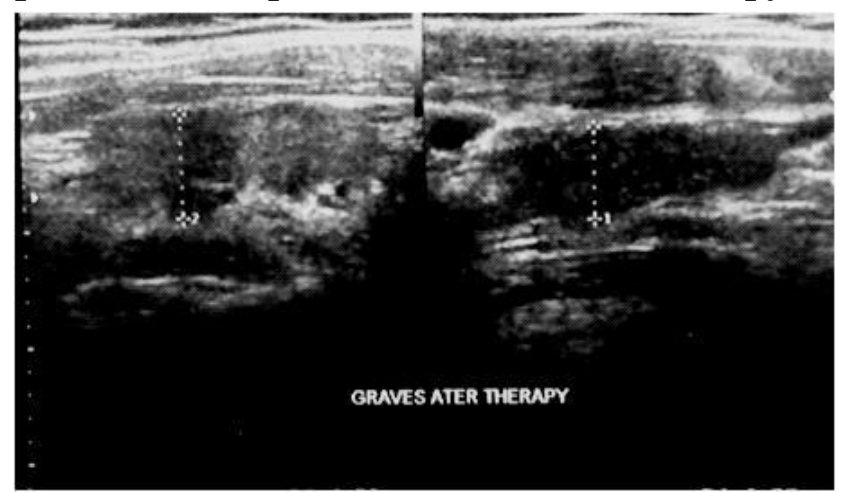

Figure 2: Revealed sizes and echogenicity of thyroid gland after radioiodine therapy of a patient of Group $B$.

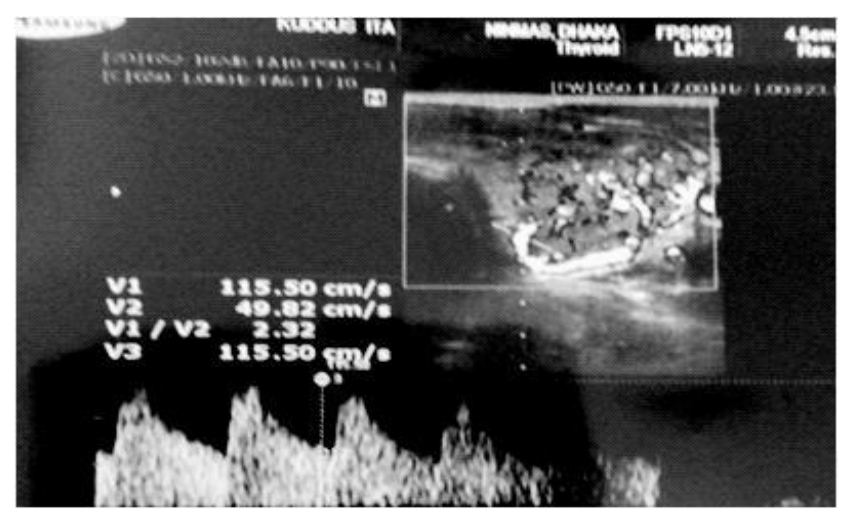

Figure 3: PSV of ITA $(115 \mathrm{~cm} / \mathrm{sec})$ of a patient in Group A.

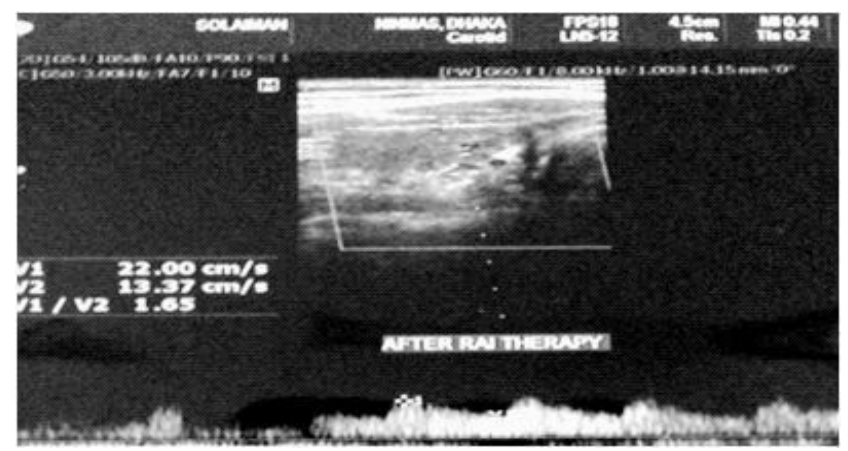

Figure 4: PSV of ITA $(22 \mathrm{~cm} / \mathrm{sec})$ 8months after radioiodine therapy of a patient in Group B .
Patients with hyperthyroidism in Group A had higher PSV values than Group B patients. Treated patients with diffuse toxic (Group B), showed a significantly lower vascularity than Group A.

Table 1: Demographic characteristics and outcome of radioiodine therapy assessed by B- mode and color Doppler Ultrasound compare with control group.

\begin{tabular}{llll}
\hline & & & $\tau$ Group C \\
\hline No. of patients & 40 & 40 & 10 \\
Male : Female & $1: 3$ & $1: 3$ & $3: 2$ \\
Age Range: & $20-65$ years & & $23-45$ years \\
AP diameter & $38=11.2 \mathrm{~mm}$ & $20 \pm 3.5 \mathrm{~mm}$ & $10=3.5 \mathrm{~mm}$ \\
PSV $(\mathrm{cm} / \mathrm{sec})$ & $70.5 \pm 15.5 \mathrm{~cm} / \mathrm{sec}$ & $22.2 \pm 12.9 \mathrm{~cm} / \mathrm{s}$ & $17.7 \pm 3.2 \mathrm{~cm} / \mathrm{s}$
\end{tabular}

"Group A: before radioiodine therapy,

**Group B: After radioiodine therapy, $\tau$ Group C: healthy volunteer

\section{DISCUSSION}

Before radioiodine therapy patients with diffuse toxic goiter (DTG) had hypervascularity of the gland as demonstrated by increased parenchymal vascularity and PSV of ITA. Ota et al. and other authors also reported similar findings of increased blood flow in untreated hyperthyroidism (8). Kurita et al after studying 75 patients with thyrotoxicosis gave a sensitivity of $84 \%$ and specificity of $90 \%$ for CDS in the diagnosis of thyrotoxicosis (9). Thus, CDS findings were comparable to nuclear imaging in the diagnosis of DTG. The sensitivity of CDS is further improved with newer methods like high resolution power Doppler for better etiological differentiation (10). Hence, CDS could be helpful in the initial evaluation of thyrotoxicosis of various etiologies and may avoid nuclear imaging in a substantial number of patients $(9,10)$.

Thus the vascularity of the thyroid gland correlates with the underlying functional status and decreases with disease under control. Ralls et al. studied patients with DTG prospectively and observed that vascularity remission occurred in parallel to biochemical remission and disease control (11). They proposed that CDS has potential to monitor therapeutic response in DTG patients without expensive laboratory tests. Therefore, 
to summarize, CDS parameters correlate well with underlying disease activity and therapy response. However, Baldiniet al. in their study demonstrated that thyroid vascularity is related more to the under-lying autoimmunity than the circulating thyroid hormone levels in euthyroid patients on therapy (12). Ten healthy volunteers were also included in this study. PSV of ITA of those volunteers was found lower than the mentioned normal reference range in other study (2). Further study with large study population is recommended to establish normal range of PSV of ITA of euthyroid persons and as well as to set up the PSV of ITA in DTG at the time diagnosis.

This preliminary data suggest that quantification of thyroid blood flow (TBF) by means of CDS might be a useful tool to predict the outcome of DTG following radioiodine therapy.

\section{CONCLUSION}

In summary, the blood flow is a useful marker in initial diagnosis of diffuse toxic goiter. The high correlation between radioisotope scan and CDS establishes this modality as an acceptable alternative. Its role in pregnancy and lactation where nuclear imaging is contraindicated needs to be emphasized. CDS evaluation of the thyroid gland is recommended as an essential part in management of diffuse toxic goiter patients especially in pregnancy.

\section{REFERENCES}

1. Bartalena, L., Diagnosis and management of Graves's disease: a global overview. Nature Reviews Endocrinology 2013;9(12):724734.

2. Ahmed Fathy Abdel Ghany and Mohamed Amin Nassef. Correlation between colour Duplex and Tc99m thyroid scan in identifying the cause of Toxic goitre. International Journal of Nuclear Medicine Research 2014;(1):7-12.

3. Ragab Hani Donkol, Aml Mohamed Nada, Sami Boughattas. Role of color Doppler in differentiation of Graves' disease and thyroiditis in thyrotoxicosis. World J Radiol 2013;5(4):178-183.

4. Bogazzi, F. and P. Vitti, Could improve ultrasound and power Doppler replace thyroidal radioiodine uptake to assess thyroid disease? Nature Clinical Practice Endocrinology \& Metabolism 2008;4(2):70-71.

5. Burch, H.B., K.D. Burman, and D.S. Cooper, A 2011 survey of clinical practice patterns in the management of Graves' disease. J Clin Endocrinol Metab 2012;(97):4549-58.

6. Andrea, F. and V. Roberto, Challenges in Neck ultrasonography: Lymph-adenopathy and Parathyroid glands. Endocrine Practice 2004;10(3):261-268.

7. Erdogan, M.F., et. al. Color flow Doppler sonography for the etiologic diagnosis of hyperthyroidism. Thyroid 2007;17:223-8.

8. Ota H, Amino N, Morita $\mathrm{S}$ et al. Quantitative measurement of thyroid blood flow for differentiation of painless thyroiditis from Graves' disease. Clin Endocrinol (Oxf) 2007;67:41-5.

9. Kurita, S., et. al. Measurement of thyroid blood flow area is useful for diagnosing the cause of thyrotoxicosis. Thyroid 2005;15(11):1249-52.

10. Mandel, S.J. Diagnostic use of ultrasonography in patients with nodular thyroid disease. Endocrine Practice 2004;10(3):246-252.

11. Ralls PW, Mayekawa DS, Lee KP, Colletti PM, Radin DR, Bosnell WD et al. Color-flow Doppler sonography in Graves' disease: 'thyroid inferno' Am J Roentgenol 1988;150:781-4.

12. Baldini M, Castagnone D, Rivolta R, Meroni L, Pappalettera M, Cantalamessa L. Thyroid vascularization by color doppler ultrasonography in Graves' disease. Changes related to different phases and to the long-term outcome of the disease. Thyroid 1997; 7:823-8. 